\title{
Mapping the Trends in Esophageal Cancer Disease Burden in the United States: Results from the Global Burden of Disease Study 2017
}

Di Lu

Department of Thoracic Surgery, Nanfang Hospital, Southern Medical University Jintao Zhan

Department of Thoracic Surgery, Nanfang Hospital, Southern Medical University

Xiguang Liu

Department of Thoracic Surgery, Nanfang Hospital, Southern Medical University

\section{Xiaoying Dong}

Department of Thoracic Surgery, Nanfang Hospital, Southern Medical University

\section{Siyang Feng}

Department of Thoracic Surgery, Nanfang Hospital, Southern Medical University Jianxue Zhai

Department of Thoracic Surgery, Nanfang Hospital, Southern Medical University

Shijie Mai

Department of Thoracic Surgery, Nanfang Hospital, Southern Medical University Jianjun Jiang

Department of Thoracic Surgery, Nanfang Hospital, Southern Medical University

\section{Zhizhi Wang}

Department of Thoracic Surgery, Nanfang Hospital, Southern Medical University

\section{Xiuyu Ji}

Department of Thoracic Surgery, Nanfang Hospital, Southern Medical University

\section{Mei Li}

Department of Thoracic Surgery, Nanfang Hospital, Southern Medical University

\section{Hua Wu}

Department of Thoracic Surgery, Nanfang Hospital, Southern Medical University

\section{Kaican Cai ( $\nabla$ doc_cai@163.com )}

Department of Thoracic Surgery, Nanfang Hospital, Southern Medical University https://orcid.org/0000-0003-4664-6694

\section{Research}


Keywords: epidemiology, burden, esophageal cancer, Global Burden of Disease, estimated annual percentage change

Posted Date: January 24th, 2020

DOl: https://doi.org/10.21203/rs.2.21803/v1

License: (c) (i) This work is licensed under a Creative Commons Attribution 4.0 International License. Read Full License 
Mapping the Trends in Esophageal Cancer Disease Burden in the United States: Results from the Global Burden of Disease Study 2017

1 Di Lu\#1, Jintao Zhan\#1, Xiguang Liu\#1, Xiaoying Dong1, Siyang Feng1, Jianxue Zhaiı, Shijie

2 Mai1, Jianjun Jiang1, Zhizhi Wang1, Xiuyu Ji1, Mei Li1, Hua Wu1, Kaican Cai1*

3 \# The authors contributed to this study equally.

4 Department of Thoracic Surgery, Nanfang Hospital, Southern Medical University, Guangzhou, 5 China.

6 * Correspondence:

$7 \quad$ Kaican Cai

8 doc_cai@163.com 


\section{Abstract}

19 Background: Esophageal cancer is the 7th leading cancer globally and the 10th leading cancer in the

20 United States. However, it is has received limited attention over more common malignancies. Only a

21 few studies have comprehensively assessed disease burden from esophageal cancer in the United

22 States (US).

23 Methods: Using states-categorized data on incidence, mortality, and Disability-adjusted Life Years

24 (DALYs), this study analyzed the current trends in esophageal cancer disease burden. Data and risk

25 factor indicators were obtained from Global Burden of Disease (GBD) online resource and used to

26 determine annual relative change.

27 Results: We report here that between 1990 and 2017, the number of esophageal cancer new cases,

28 deaths and DALYs in the US increased significantly, while the Age-standardized Rate (ASR) of

29 disease incidence remained constant. During the same time, disease burden from esophageal cancer

30 in males was higher than that in females. Economically stronger states trend to had lesser disease

31 burden from esophageal cancer. Smoking and alcohol use contributed most of the burden while

32 influence of high body-mass index and diet low in fruits grew largely.

33 Conclusions: This study provided an analysis of esophageal cancer disease burden in the United

34 States that will inform the design of targeted strategies for disease prevention tailored to different 35 states.

36 Keywords: epidemiology, burden, esophageal cancer, Global Burden of Disease, estimated

37 annual percentage change 
40 Esophageal cancer, the 7 th leading cancer globally, is becoming the fastest growing cancer in the

41 Western world. In the United States, esophageal cancer is now the $10_{\text {th }}$ leading cancer overall, and the

427 th leading cause of cancer deaths in males. The disease contributes to $20 \%$ increase in US male death

43 rates and approximately $85 \%$ country mortality rate. Despite of this, esophageal cancer still receive

44 little attention from government agencies and the research community, with a negative implication on

45 establishment of a large-scale public health intervention[1-3].

46 Previous studies have reported regional, temporal and ethnic differences in esophageal cancer

47 disease burden in United States[4-6]. However, most of them employ incidence as the sole parameter

48 for analyzing disease burden. Only a few have reported using multiple parameters and datasets to study

49 esophageal cancer disease burden in the US.

50 The Global Burden of Disease (GBD) study is published by the Global Health Data Exchange

51 (GHDx), world's most comprehensive catalog of surveys, censuses, vital statistics, and other health-

52 related data. Additionally, GHDx is unique in its approach for generating estimates for all locations

53 using all available data from literature, administrative hospital and medical claims records, and cause

54 of death records[7, 8]. GBD provides multi-angle epidemiological data on different diseases from

55 different locations.

56 This study used GBD data to study disease burden from esophageal cancer in the United States under

57 three parameters; incidence, mortality, Disability-adjusted Life Years (DALYs) in an effort to provide

58 a comprehensive view of esophageal cancer. In addition, esophageal cancer disease burden attributed

59 to different risk factors in different states was studied, and association between burden and the

60 respective economic status explored. 


\section{Methods}

\section{Incidence, mortality and DALYs estimates}

64 Incidence, mortality and DALYs for esophageal cancer in the United States between 1990-2017 was

65 obtained from the GBD online repository. Accessed data had been categorized by gender, year, measure, 66 metric, age, location and risk factors.

67 DALYs is a metric describing the total time lost due to a health condition, including premature death 68 and disability. The metric was developed by the World Health Organization (WHO) GBD study of the 69 year 1990 and has been considered a standard measure of disease burden since then[7, 9].

\section{Age-standardized rate and estimated annual percentage change}

71 Age-standardized rate (ASR) and estimated annual percentage change (EAPC) were used to 72 analyze the burden from esophageal cancer. Age structures of population in different states are not 73 similar and changes over time. This necessitates age standardization to limit the effect of difference 74 age structures on disease burden. ASR of incidence, mortality and disability-adjusted life years were 75 acquired from the GBD.

76 Annual relative change is a method to evaluate trends in age-adjusted rates, obtained from a log$77 \quad$ liner modal as

$$
\log R=\beta_{0}+\beta_{1}+\varepsilon
$$

79 where $R=$ age-adjusted rate, and $X=$ calendar year. Annual percent change is given by $100 \times\left[\exp \left(\beta_{1}\right)\right)$ -

$801][10-13]$. Age-standardized rate was presented as ASR (obtained from $\ln R=\beta_{0}+\beta_{1} X+\varepsilon$ ), and EAPC 81 of ASR was calculated as $\left.100 \times\left[\exp \left(\beta_{1}\right)\right)-1\right]$. The ASR was considered to be increasing if the EAPC 82 estimation and the lower boundary of its $95 \%$ Confidence Interval (CI) were both greater than 0 . In 83 contrast, the ASR was considered to be decreasing if the EAPC estimation and the upper boundary of 
84 its 95\% CI were both lesser than 0. Otherwise, ASR was considered stable over the period.

\section{Per capita disposable personal income}

86 To explore the relation between the economic level and burden from esophageal cancer, a linear

87 regression model was drawn for per capita disposable personal income (PCDPI) and ASR or EAPC of 88 incidence, mortality and DALYs.

\section{Statistical analysis}

90 Data was analyzed using the IBM SPSS statistics version 23, and graphs drawn using GraphPad 91 Prism 8 and Microsoft Excel (Office 365).

93 Results

\section{Incidence}

95 New cases of esophageal cancer in the United States increased from $11391.02 \times 1000$ in the year 961990 to $20690.21 \times 1000$ in 2017. Although the age-standardized rate of esophageal cancer in 2017

97 (3.85 per 100,000) was higher than that of 1990 (3.61 per 100,000), there was no significance difference

98 in the estimated annual percentage change. In both 1990 and 2017, the US male population contributed

99 four-fold more incidences of new esophageal cancer cases over females (Table 1). However, between

100 1990-2017, the age-standardized rate of esophageal cancer incidences in males remained constant

101 while it decreased in females with an estimated annual percentage change of $-0.444 \%$ (Table 1 ).

102 At states level, between 1990 and 2017, the highest increase of new esophageal cancer cases was 103 recorded in Nevada (262.32\%), followed by Alaska (246.51\%) and Utah (208.88\%). In contrast, the 104 lowest increase of new cases was observed in New York (30.83\%), followed by New Jersey (39.02\%) 105 and Michigan (49.40\%). In the District of Columbia, emergence of new cases decreased by $-24.84 \%$ 
106 (Figure 1A). The highest age-standardized rate of incidence in 2017 was recorded in South Dakota

107 (5.93 per 100,000), followed by Colorado (5.40 per 100,000) and Virginia (5.17 per 100,000). On the 108 other hand, the lowest age-standardized rate of incidence in 2017 was recorded in Oklahoma (2.88 per

109 100,000), followed by Montana (3.03 per 100,000) and Kentucky (3.15 per 100,000) (Figure 1B).

110 Between 1990-2017, the largest increase in ASR of incidence was observed in Oklahoma $($ EAPC $=$

$1111.301 \%)$, followed by West Virginia $($ EAPC $=1.187 \%)$ and Arkansas $($ EAPC $=0.999 \%)$, while the

112 largest decrease was observed in the District of Columbia (EAPC $=-2.112 \%$ ), followed by Maryland

$113($ EAPC $=-0.900 \%)$ and California $($ EAPC $=-0.786 \%)$. In the same time, 6 states recorded a constant

114 ASR of incidence (Figure 1C). A significant negative correlation was observed between EAPC of

115 incidence and PCDPI in 1990 (Figure 1D), while there was no significant correlation between ASR of

116 incidence and PCDPI in 2017 (Figure 1E).

\section{Mortality}

118 Deaths from esophageal cancer in the United States rose from $11391.02 \times 1000$ in 1990 to 15097.64

$119 \times 1000$ in 2017. Nonetheless, in the same period, the ASR of mortality decreased from 2.91 per 100,000

120 to 2.79 per 100,000 with an average $-0.347 \%$ per year change. In both 1990 and 2017 , males

121 contributed the majority of deaths recorded, with a quintuple higher ASR of mortality over females.

122 However, the ASR of mortality decreased in both males and females, with an EAPC of $-0.340 \%$ and -

$1231.116 \%$, respectively. Smoking was the leading risk factor in esophageal cancer mortality, followed by

124 high body-mass index, alcohol consumption, low-fruit diet and chewing tobacco. Nonetheless, the 125 fastest growing risk factor was high body-mass index $(\mathrm{EAPC}=0.777 \%)$, followed by low-fruit diet

$126(\mathrm{EAPC}=0.547 \%)$. Risk factor contribution by alcohol consumption remained constant while smoking

$127($ EAPC $=-1.613 \%)$ and chewing tobacco $($ EAPC $=-0.481 \%)$ decreased over time (Table 2$)$.

128 The highest increased in deaths was observed in Nevada (236.67\%), followed by Alaska (215.58\%) 
129 and Arizona (174.93\%), while the lowest was observed in New York (11.15\%), followed by New Jersey

$130(19.63 \%)$ and Illinois (30.47\%). District of Columbia was the only state recording a decrease in deaths

$131(-34.75 \%)$ (Figure 2A). In 2017, the highest ASRs of mortality was recorded in District of Columbia

132 (4.49 per 100,000), followed by Maine (3.79 per 100,000) and Ohio (3.60 per 100,000), while the

133 lowest one was observed in Utah (1.95 per 100,000) followed by Hawaii $(2.05$ per 100,000) and

134 California (2.12 per 100,000) (Figure 2B). Between 1990-2017, the District of Columbia had the

135 highest ASR of mortality. Furthermore, its ASR of mortality recorded the fastest decrease $($ EAPC $=$ -

$1362.591 \%)$, followed by Maryland $(\mathrm{EAPC}=-1.325 \%)$ and New York $(\mathrm{EAPC}=-1.048 \%)$. The fastest

137 growing ASR of mortality was recorded in Oklahoma $($ EAPC $=1.142 \%)$, followed by West Virginia

$138(\mathrm{EAPC}=0.920 \%)$ and Arkansas $(\mathrm{EAPC}=0.723 \%)$. Fourteen states sustained a constant ASR of

139 mortality (Figure 2C). Moreover, a strong correlation was observed between PCDPI and EAPC of

140 mortality that was not apparent in the ASR of mortality (Figure 2D, 2E).

141 In 2017, the highest contribution of risk factors (smoking, 2.33 per 100,000; alcohol use, 2.12 per

142 100,000; high body-mass index, 2.16 per 100,000; and low-fruit diet, 0.75 per 100,000) to the ASR of

143 mortality was recorded in the District of Columbia. Chewing tobacco was the major risk factor in West

144 Virginia (0.66 per 100,000). The investigated risk factors had the highest impact on the ASR of 145 mortality in the District of Columbia, Maine and Ohio (Figure 3A). The highest increase in ASR of 146 mortality resulting from alcohol use, high body-mass index and low-fruit diet was observed in 147 Oklahoma with an EAPC of $1.495 \%, 2.612 \%$ and $2.229 \%$, respectively. In Arkansas, chewing tobacco 148 was a significant risk factor with an EAPC of $1.535 \%$. Decrease in ASR of mortality as a result of 149 smoking, alcohol use, high body-mass index and chewing tobacco was observed in the District of 150 Columbia with an EAPC of $-3.815 \%,-2.365 \%,-1.892 \%$ and -2.171 , respectively. In California, low151 fruit diet was the least contributing risk factor with an EAPC of $-0.906 \%$. Overall, the ASR of mortality 152 attributed to smoking declined in all states except Oklahoma (with no significant EAPC). In contrast, 
153 the ASR of mortality attributed to high body-mass index and low-fruit diet increased in majority of

154 sates as shown in Figure 3B. PCDPI was negatively correlated with ASR of mortality attributed to 155 smoking, low-fruit diet and chewing tobacco (Figure 3C-G), and that of EAPC of mortality attributed

156 to all risk factor investigated except for chewing tobacco (Figure 6H-L).

\section{DALYs}

158 Disability-adjusted life years of esophageal cancer in United States increased from 213467.95 years 159 in 1990 to 330723.23 years in 2017. Additionally, the ASR of DALYs decreased from 70.92 years per 160100,000 to 64.18 years per 100,000 in the same period with an average $-1.238 \%$ per year change. In 161 both 1990 and 2017, males made the largest contribution to DALYs and ASR of DALYs. However, a 162 decreasing trend was recorded in the ASRs of DALYs for both males (EAPC $=-0.576 \%$ ) and females 163 (EAPC $=-0.563 \%)$. The most significant risk factor attributed to DALYs was smoking, while the

164 largest ASR of DALYs was recorded in alcohol use, followed by high-body mass index, smoking, diet 165 low in fruits and chewing tobacco. The ASR of DALYs contributable to smoking (EAPC $=-1.912 \%$ ) 166 recorded the fasted dip, followed by chewing tobacco $($ EAPC $=-0.627 \%)$ and alcohol use $($ EAPC $=$ 167 0.197\%). In contrast, change in DALYs and ASR of DALYs attributed to high body-mass index and 168 low-fruit diet increased with an EAPC of $0.510 \%$ and $0.280 \%$, respectively (Table 3 ).

169 The highest increase in DALYs was observed in Nevada (67.54\%), followed by Alaska (64.65\%) 170 and Utah (62.67\%) while the lowest increase was recorded in New York (0.72\%), New Jersey $(10.12 \%)$ 171 and Illinois (18.51\%). The district of Columbia was the only state recording a decrease in deaths from 172 esophageal cancer (-63.69\%) (Figure 4A). In 2017, the highest ASR of DALYs was observed in District 173 of Columbia (108.65 years per 100,000), followed by Maine (86.85 years per 100,000) and West

174 Virginia (84.38 years per 100,000), while the lowest ASR was observed in Utah (44.92 years per 175 100,000), followed by California (46.64 years per 100,000) and Hawaii (49.12 years per 100,000) 176 (Figure 4B). Although the ASR of mortality was highest in the District of Columbia, the state recorded 
177 a rapid dip in ASR of DALYs between 1990-2017 with an EAPC of -2.879\%. This was closely followed

178 by Maryland $(\mathrm{EAPC}=-1.575 \%)$ and New York $(\mathrm{EAPC}=-1.456 \%)$. The highest increase in ASR of

179 DALYs was observed in Oklahoma $($ EAPC $=1.109 \%)$, followed by West Virginia $($ EAPC $=1.013 \%)$

180 and Arkansas $($ EAPC $=0.709 \%)$. Ten states sustained a constant ASR of DALYs (Figure 4C). There

181 was a significant association between PCDPI and EAPC of mortality that was not apparent in ASR of 182 mortality (Figure 4D, 4E).

183 In 2017, the highest ASR of DALYs attributed to smoking, alcohol use, high body-mass index and 184 low-fruit diet was observed in the District of Columbia $(53.57$ years per 100,000, 53.99 years per $185100,000,53.08$ years per 100,000, 18.40 years per 100,000, respectively), while that attributed to 186 chewing tobacco was recorded in West Virginia (15.92 years per 100,000). In the states of District of 187 Columbia, Maine, West Virginia and Ohio, high ASRs of DALYs was attributed to the investigated risk 188 factors (Figure 5A). The highest increase in ASR of DALYs as a result of high body-mass index and 189 low-fruit diet was recorded in Oklahoma (EAPC $=2.505 \%$ and $2.186 \%$, respectively), while that 190 attributed to alcohol use was observed in West Virginia (EAPC $=1.462 \%)$ and chewing tobacco in 191 Arkansas $(\mathrm{EAPC}=1.556 \%)$. In contrast, the most significant decrease in ASRs of DALYs attributed 192 to smoking, alcohol use, high body-mass index and chewing tobacco was recorded in the District of 193 Columbia (EAPC $-4.178 \%,-2.668 \%,-2.195 \%$ and $-2.503 \%$, respectively), while that of low-fruit diet 194 was observed in California $($ EAPC $=-1.276 \%$ ). There was a decrease in ASR of DALYs attributed to smoking in all states except Oklahoma (with an insignificant EAPC). However, ASR of DALYs 196 attributed to high body-mass index and low-fruit diet increased in most states (Figure 5B). A negative 197 correlation was observed between PCDPI and ASR of DALYs as a result of smoking, low-fruit diet 198 and chewing tobacco (Figure 5C-G), as well as the EAPC of DALYs attributed to all risk factor except 199 chewing tobacco (Figure 5H-L). 


\section{Discussion}

202 This study investigated disease burden from esophageal cancer under the parameters of incidence, 203 mortality as well as DALYs. Findings presented an overview of disease burden in different states and

204 the contribution of selected risk factors. Results from this study suggested that incidence rate of 205 esophageal cancer in United States has kept stable during 1990 to 2017 while mortality rate and DALYs 206 rate kept decreasing. During the same time, disease burden from esophageal cancer in males was higher 207 than that in females. Additionally, states that were economically stronger had a lesser disease burden 208 from esophageal cancer. Smoking was the most significant risk factor contributing to disease burden 209 but high body-mass index and diet low in fruits played more and more important roles.

210 Studies show that esophageal adenocarcinoma (EA) has increased in the United States while

211 esophageal squamous cell carcinoma (ESCC) is continually decreasing $[5,14,15]$. Studying different

212 histological types as a whole, it was suggested in our study that respiting largely growing new cases,

213 incidence rate of esophageal cancer in United States has kept stable during 1990 to 2017. During the

214 same time, mortality rate and DALYs rate of esophageal cancer in United States decreased, though the

215 total number of deaths and DALYs increased significantly. As reported by Global Burden of Disease

216 Study 2017 (GBD 2017) Population Estimates 1950-2017, population in United States had risen from

2172.534 billion in 1990 to 3.248 billion in 2017 . This might be the reason why new cases, deaths and

218 DALYs of esophageal cancer all rose enormously though incidence rate kept stable while mortality

219 rate and DALYs rate decreased. The recorded declining disease burden from esophageal cancer in

220 United States indicated that control strategies put in place have been effective.

221 Nicolas Patel and Bikramjit Benipal reported a higher incidence of esophageal cancer in males 222 compared to females in the United States[5] which was similar to the report by Luckson N et al.[16].

223 From this study, disease burden from esophageal cancer in males was higher than that in females, with

224 significantly larger ASRs of incidence, mortality and DALYs. Overall, we report a general decrease in 
225 disease burden from esophageal cancer in both the male and female population, which was more 226 obvious in female population in incidence rate and mortality rate. This difference in disease burden 227 from esophageal cancer suggested that female might be a protective factor for esophageal cancer.

228 Females were in less risk of esophageal cancer, which might be resulted by different endocrine milieu

229 in males and females. Studies had reported the effect of Estrogen in inhibiting esophageal squamous

230 cell cancer growth both in vitro[17, 18] and in vivo[19]. The estrogen receptor ER beta was thought to

231 be responsible to this anti-proliferative effect, which had reported to be expressed also in esophageal

232 adenocarcinoma[20]. On the other hand, better prognosis was observed in females, as results of

233 different endocrine milieu[21], lower alcohol consumption and smoking addiction[22], different gene

234 expression[23] and so on.

235 Jennifer Drahos et al. reported significant geographical variability in esophageal adenocarcinoma 236 incidence rate and esophageal squamous cell carcinoma incidence rate by census region (the Northeast,

237 Midwest, South, and West)[6]. From this study, although in average the ASR of incidence did not 238 change over time while the ASR of mortality and DALYs decreased, all three parameters increased in 239 the states of Oklahoma, West Virginia, Arkansas, North Dakota, Iowa, Kansas, Indiana, Kentucky, 240 South Dakota, Utah, Idaho, Wyoming, Maine, Ohio, Nebraska and Tennessee. Interestingly, in coastal 241 area states, ASR of incidence, mortality and DALYs recorded a decreasing trend. It seemed this 242 geographical variability might to some extent be resulted by economical variability in different states.

243 To explain why geographical variability was observed in disease burden from esophageal cancer, we 244 explored the association between economic level and disease burden from esophageal cancer. Findings 245 indicated that states that were economically stronger had a lesser disease burden from esophageal 246 cancer. This was particularly apparent in the burden attributed to smoking, alcohol use, high body-mass 247 index or diet low in fruits. Economic factor plays an important part in incidence rate and mortality rate 248 of esophageal cancer. Low socioeconomical status was in relation to the higher incidence, which might 
be a result of combined influence of more alcohol use and smoking, lower annual income, lower annual

250 expenditure on food, lower annual expenditure on fruit and vegetables, higher percentage of

251 unemployment, and higher percentage of employment in agriculture and construction sectors and so

252 on[24-26]. In addition, low socioeconomical status was in relation to poorer prognosis, resulted by

253 poorer cognition of the malignant disease, poorer access to health services, less performed resection

254 and chemotherapy and so on[27-29].

255 Numerous risk factors contribute to esophageal cancer[3, 9, 15, 30-34]. GBD lists smoking, alcohol

256 use, high body-mass index, diet low in fruits and chewing tobacco as the five main risk factors

257 associated with esophageal cancer. From our analysis, smoking was the most significant risk factor

258 contributing to disease burden from esophageal cancer in United Sates. In most states, the effect of

259 alcohol use and chewing tobacco either did not vary or reduced over time. However, high body-mass

260 index and diet low in fruits played more and more important roles, with the disease burden from

261 esophageal cancer attributed increasing in most states. The change of population with different factors

262 might explain the trend of disease burden from esophageal cancer attributed to different factors to a

263 certain degree. Data from The Behavioral Risk Factor Surveillance System (BRFSS) reported that the

264 population with smoking had decreased from 22.4\% in 1995 to $17.1 \%$ in 2017, while alcohol use from

$26552.8 \%$ in 1995 to $54.7 \%$ in 2017 , high body-mass index from $52.0 \%$ in 1995 to $66.2 \%$ in 2017 ,

266 Chewing tobacco from 4.3\% in 2013 to $4.0 \%$ in 2017(It was not recommended by BRFSS to compare

267 fruit and vegetable intake from 2017 to prior years due to the changes in methodology). However, there

268 were still some doubts not settled. For example, why smoking was the most significant risk factor

269 though its related population was not the largest? Why were the trends of mortality rate and DALYs

270 rate contributed to diet low in fruits in conflict with the change of population who with low-fruits diets?

271 A possible explain was that the effects of different risk factors on prognosis of esophageal cancer were

272 different, but the deeper reason needed further explore. 
273 This study had a few limitations that constrained the range of insights generated from our analysis.

274 First, GBD online resource lack data on different histological types of esophageal cancer, thereby

275 limiting our ability to compare disease burden between histological types. Secondly, it was not feasible

276 distinguishing whether a person was affected by one or more risk factors, thereby missing the 277 opportunity to analyze the interactions among different risk factors and their influence on disease

278 burden from esophageal cancer. Thirdly, datasets from the GBD are estimates of quantified health loss.

279 Therefore, accuracy of the results is as good as the data collected and shared by the GBD consortium.

\section{Conclusions}

282 We provide here trends in esophageal cancer burden in the United States that will inform design of 283 efficient prevention strategies tailored for different states. A recommendation is fronted for the United 284 States to strengthen efforts in controlling smoking while encouraging and supporting optimal body285 mass index and inclusion of fruits in diets. Ultimately, economically weaker states (Oklahoma, West 286 Virginia and so on, in which ASRs of incidence, mortality and DALYs all increased) should make 287 deliberate efforts to contain the growing disease burden from esophageal cancer.

289 List of abbreviations

$290 \quad$ GBD Global Burden of Disease

291 DALYs Disability-adjusted Life Years

292 PCDPI Per Capita Disposable Personal Income

293 ASR Age-standardized Rate 
296 Declarations

297 Ethics approval and consent to participate

298 Not applicable

299 Consent for publication

300 Not applicable

301 Availability of data and materials

302 All data was obtained from the GBD online resource available at http://ghdx.healthdata.org. PCDPI

303 of 50 states and the District of Columbia between 1990-2017 was obtained from the Bureau of Labor

304 Statistics available at https://www.bls.gov/.

305 Competing interests

306 The authors alone are responsible for the views expressed in this article and they do not necessarily

307 represent the views, decisions, or policies of the institutions with which they are affiliated. The

308 authors declare that they have no competing interest.

309 Funding

310 This study was financially supported by the Science and Technology Planning Project of Guangdong

311 Province [2017B020226005] and the Undergraduate Innovative and Entrepreneurial Training

312 Program of Guangdong Prrovince [201812121156]. 
314 DL, JZ (Jintao Zhan) and KC conceptualized the study. DL, JZ (Jintao Zhan) and XL designed the

315 study. JZ (Jintao Zhan) and XL acquired the epidemiology / PCDPI data. DL, JZ (Jintao Zhan), XL

316 and XD analyzed the data. SF, JZ (Jianxue Zhai) and SM drew the statistic figures and tables. JJ, ZW

317 and XJ reexamined the results. DL and JZ (Jintao Zhan) wrote the first draft of the manuscript. ML,

318 HW and KC revised the manuscript. All authors reviewed the manuscript and approved the final

319 version of the manuscript.

320 Acknowledgements

321 We gratefully thank all GBD Study participants and staff for their time and commitment to the study.

Reference

1. Chai J, Jamal MM: Esophageal malignancy: a growing concern. World J Gastroenterol 2012, 18:6521-6526.

2. Zhang Y: Epidemiology of esophageal cancer. World J Gastroenterol 2013, 19:5598-5606.

3. Palladino-Davis AG, Mendez BM, Fisichella PM, Davis CS: Dietary habits and esophageal cancer. Dis Esophagus 2015, 28:59-67.

4. Kubo A, Corley DA: Marked regional variation in adenocarcinomas of the esophagus and the gastric cardia in the United States. Cancer 2002, 95:2096-2102.

5. Patel N, Benipal B: Incidence of Esophageal Cancer in the United States from 2001-2015: A United States Cancer Statistics Analysis of 50 States. Cureus 2018, 10:e3709.

6. Drahos J, Wu M, Anderson WF, Trivers KF, King J, Rosenberg PS, Eheman C, Cook MB: Regional variations in esophageal cancer rates by census region in the United States, 19992008. PLoS One 2013, 8:e67913.

7. Collaborators GBDS: Global, regional, and national burden of stroke, 1990-2016: a systematic analysis for the Global Burden of Disease Study 2016. Lancet Neurol 2019, 18:439-458.

8. Zhang X, Yao ZQ, Karuna T, He XY, Wang XM, Li XF, Liu WC, Li R, Guo SQ, Chen YC, et al: The role of wall shear stress in the parent artery as an independent variable in the formation status of anterior communicating artery aneurysms. European Radiology 2019, 29:689-698.

9. Di Pardo BJ, Bronson NW, Diggs BS, Thomas CR, Jr., Hunter JG, Dolan JP: The Global Burden of Esophageal Cancer: A Disability-Adjusted Life-Year Approach. World J Surg 2016, 40:395-401. 
10. Hankey BF, Ries LA, Kosary CL, Feuer EJ, Merrill RM, Clegg LX, Edwards BK: Partitioning

11. Cole P, Rodu B: Declining cancer mortality in the United States. Cancer 1996, 78:20452048.

12. DM E: A computer-based model for designing cancer control strategies. Natl Cancer Inst Monogr 1986:75-82.

13. EL L: Elements of Large-Sample Theory. New York 1999.

14. Kim JY, Winters JK, Kim J, Bernstein L, Raz D, Gomez SL: Birthplace and esophageal cancer incidence patterns among Asian-Americans. Dis Esophagus 2016, 29:99-104.

15. Blot WJ, McLaughlin JK: The changing epidemiology of esophageal cancer. Semin Oncol 1999, 26:2-8.

16. Mathieu LN, Kanarek NF, Tsai HL, Rudin CM, Brock MV: Age and sex differences in the incidence of esophageal adenocarcinoma: results from the Surveillance, Epidemiology, and End Results (SEER) Registry (1973-2008). Dis Esophagus 2014, 27:757-763.

17. Matsuoka H, Sugimachi K, Ueo H, Kuwano H, Nakano S, Nakayama M: Sex hormone response of a newly established squamous cell line derived from clinical esophageal carcinoma. Cancer Res 1987, 47:4134-4140.

18. Ueo H, Matsuoka H, Sugimachi K, Kuwano H, Mori M, Akiyoshi T: Inhibitory effects of estrogen on the growth of a human esophageal carcinoma cell line. Cancer Res 1990, 50:7212-7215.

19. Utsumi Y, Nakamura T, Nagasue N, Kubota H, Morikawa S: Role of estrogen receptors in the growth of human esophageal carcinoma. Cancer 1989, 64:88-93.

20. Hennessy BA, Harvey BJ, Healy V: 17beta-Estradiol rapidly stimulates c-fos expression via the MAPK pathway in T84 cells. Mol Cell Endocrinol 2005, 229:39-47.

21. Badwe RA, Patil PK, Bhansali MS, Mistry RC, Juvekar RR, Desai PB: Impact of age and sex on survival after curative resection for carcinoma of the esophagus. Cancer 1994, 74:24252429.

22. Hidaka H, Hotokezaka M, Nakashima S, Uchiyama S, Maehara N, Chijiiwa K: Sex difference in survival of patients treated by surgical resection for esophageal cancer. World J Surg 2007, 31:1982-1987.

23. Sanford NN, Mahal BA, Royce TJ, Pike LRG, Hwang WL: Sex Disparity and Copy Number Alterations in Esophageal Squamous Cell Carcinoma. Clin Gastroenterol Hepatol 2019, 17:1207-1209.

24. Brown LM HR, Silverman D, Baris D, Hayes R, Swanson GM, Schoenberg J, Greenberg R, Liff J, Schwartz A, Dosemeci M, Pottern L, Fraumeni JF Jr.: Excess incidence of squamous cell esophageal cancer among US Black men: role of social class and other risk factors. Am J Epidemiol 2001.

25. Amorim CA, De Souza LP, Moreira JP, Luiz RR, De VCAJ, De Souza HSP: Geographic distribution and time trends of esophageal cancer in Brazil from 2005 to 2015. Mol Clin Oncol 2019, 10:631-638.

26. Mohebbi M, Wolfe R, Jolley D, Forbes AB, Mahmoodi M, Burton RC: The spatial distribution of esophageal and gastric cancer in Caspian region of Iran: an ecological analysis of diet and socio-economic influences. Int J Health Geogr 2011, 10:13.

27. Wang N, Cao F, Liu F, Jia Y, Wang J, Bao C, Wang X, Song Q, Tan B, Cheng Y: The effect of socioeconomic status on health-care delay and treatment of esophageal cancer. $J$ Transl Med 2015, 13:241.

28. Schlottmann F, Gaber C, Strassle PD, Herbella FAM, Molena D, Patti MG: Disparities in esophageal cancer: less treatment, less surgical resection, and poorer survival in disadvantaged patients. Dis Esophagus 2019. 
29. Wu CC, Chang CM, Hsu TW, Lee CH, Chen JH, Huang CY, Lee CC: The effect of individual and neighborhood socioeconomic status on esophageal cancer survival in working-age patients in Taiwan. Medicine (Baltimore) 2016, 95:e4140.

30. Zhang SS, Yang H, Luo KJ, Huang QY, Chen JY, Yang F, Cai XL, Xie X, Liu QW, Bella AE, et al: The impact of body mass index on complication and survival in resected oesophageal cancer: a clinical-based cohort and meta-analysis. British Journal of Cancer 2013, 109:2894-2903.

31. Blot WJ: Esophageal cancer trends and risk factors. Semin Oncol 1994, 21:403-410.

32. Holmes RS, Vaughan TL: Epidemiology and pathogenesis of esophageal cancer. Semin Radiat Oncol 2007, 17:2-9.

33. Kamangar F, Chow WH, Abnet CC, Dawsey SM: Environmental causes of esophageal cancer. Gastroenterol Clin North Am 2009, 38:27-57, vii.

34. Domper Arnal MJ, Ferrandez Arenas A, Lanas Arbeloa A: Esophageal cancer: Risk factors, screening and endoscopic treatment in Western and Eastern countries. World $J$ Gastroenterol 2015, 21:7933-7943.

\section{Figure legends}

Figure 1. Maps of parameters of incidence, and the correlation between PCDPI and EAPC.

(A) The relative change in new cases of esophageal cancer between 1990 and 2017. (B) The ASR of incidence of esophageal cancer in 2017 (C) The EAPC of ASR of incidence of esophageal cancer from 1990 to 2017. (D)The correlation between PCDPI and ASR of incidence of esophageal cancer. (E) The correlation between PCDPI and EAPC. The B indices and $\mathrm{p}$ values presented in (D) and (E) were derived from Pearson correlation analysis. The circles in (C) to (L) represent states. ASR, agestandardized rate; EAPC, estimated annual percentage change. $\mathbf{\Lambda}$, EAPC is not significant. District of Columbia was not included in (D) and (E) because of its outlier high PCDPI.

Figure 2. Maps of parameters of mortality, and the correlation between PCDPI and EAPC.

(A) ASRs of mortality of esophageal cancer attributed to different risk factors in different states. (B) EAPC of mortality of esophageal cancer attributed to different risk factors in different states. (C-G) 
424 The correlation between PCDPI and ASR of mortality of esophageal cancer attributed to certain risk

425 factor and its EAPC. (H-L) The correlation between PCDPI and EAPC of mortality of esophageal 426 cancer attributed to certain risk factor and its EAPC. ASR, age-standardized rate; EAPC, estimated

427 annual percentage change. Boxes with slashes mean that EAPC is not significant. The circles

428 represent states. The B indices and $\mathrm{p}$ values were derived from Pearson correlation analysis. ASR, 429 age-standardized rate; EAPC, estimated annual percentage change. District of Columbia was not 430 included in (C) and (L) because of its outlier high PCDPI.

432 Figure 3. Heat map of EAPCs of mortality, and the correlation between PCDPI and EAPC.

433 (A) ASRs of mortality of esophageal cancer attributed to different risk factors in different states. (B) 434 EAPC of mortality of esophageal cancer attributed to different risk factors in different states. (C-G) 435 The correlation between PCDPI and ASR of mortality of esophageal cancer attributed to certain risk 436 factor and its EAPC. (H-L) The correlation between PCDPI and EAPC of mortality of esophageal 437 cancer attributed to certain risk factor and its EAPC. ASR, age-standardized rate; EAPC, estimated 438 annual percentage change. Boxes with slashes mean that EAPC is not significant. The circles 439 represent states. The B indices and $\mathrm{p}$ values were derived from Pearson correlation analysis. ASR, 440 age-standardized rate; EAPC, estimated annual percentage change. District of Columbia was not 441 included in (C) and (L) because of its outlier high PCDPI.

443 Figure 4. Maps of parameters of DALYs, and the correlation between PCDPI and EAPC.

444 (A) The relative change in DALYs of esophageal cancer between 1990 and 2017. (B) The ASR of 445 DALYs of esophageal cancer in 2017. (C) The EAPC of ASR of DALYs of esophageal cancer from 

1990 to 2017. (D) The correlation between PCDPI and ASR of DALYs of esophageal cancer. (E)

447 The correlation between PCDPI and EAPC. The B indices and p values presented in (D) and (E)

448 were derived from Pearson correlation analysis. The circles in $(\mathrm{C})$ to $(\mathrm{L})$ represent states. DALYs, 449 mortality and disability adjusted life years; ASR, age-standardized rate; EAPC, estimated annual

450 percentage change. $\boldsymbol{\Delta}$, EAPC is not significant. District of Columbia was not included in (D) and (E) 451 because of its outlier high PCDPI.

453 Figure 5. Heat map of EAPCs of DALYs, and the correlation between PCDPI and EAPC.

454 (A) ASRs of DALYs of esophageal cancer attributed to different risk factors in different states. (B) 455 EAPC of DALYs of esophageal cancer attributed to different risk factors in different states. (C-G) 456 The correlation between PCDPI and ASR of DALYs of esophageal cancer attributed to certain risk 457 factor and its EAPC. (H-L) The correlation between PCDPI and EAPC of DALYs rate of esophageal 458 cancer attributed to certain risk factor and its EAPC. ASR, age-standardized rate; EAPC, estimated 459 annual percentage change. Boxes with slashes mean that EAPC is not significant. The circles 460 represent states. The B indices and $\mathrm{p}$ values were derived from Pearson correlation analysis. DALYs, 461 mortality and disability adjusted life years; ASR, age-standardized rate; EAPC, estimated annual 462 percentage change. District of Columbia was not included in (C) to (L) because of its outlier high 463 PCDPI. 
466 Table 1. New cases, ASRs of incidence of esophageal cancer in 1990 and 2017 and temporal trends.

\begin{tabular}{|c|c|c|c|c|c|}
\hline \multirow[t]{2}{*}{ Category } & \multicolumn{2}{|c|}{ New cases. $\times 1000(95 \%$ UI $)$} & \multicolumn{2}{|c|}{ ASR per $100,000(95 \%$ UI) } & \multirow{2}{*}{$\begin{array}{c}\text { EAPC \% }(95 \% \text { CI }) \\
1990-2017\end{array}$} \\
\hline & 1990 & 2017 & 1990 & 2017 & \\
\hline \multirow[t]{2}{*}{ Overall } & 11391.02 & 20690.21 & 3.61 & 3.85 & 0.007 \\
\hline & $(11247.59,11556,12$ & $(20040.79,21331.42)$ & $(3.56,3.66)$ & $(3.73,3.97)$ & $(-0.186,0.200)$ \\
\hline
\end{tabular}

Sex

Male

8604.47

16415.49

6.28

6.64

$-0.006$

$(8467.84,8771.37) \quad(15778.08,17032.67)$

$(6.18,6.39)$

$(6.38,6.90)$

$(-0.197,0.186)$

Female

2786.55

4274.72

1.50

1.45

$-0.444$

$(2731.80,2843.72)$

$(4087.10,4452.67)$

$(1.47,1.53)$

$(1.39,1.51)$

$(-0.667,-0.220)$

467 ASR, age-standardized rate; CI, confidence interval; EAPC, estimated annual percentage change; UI, 468 uncertainty interval. 
471 Table 2. Deaths and ASRs of mortality of esophageal cancer in 1990 and 2017 and temporal trends.

\begin{tabular}{|c|c|c|c|c|c|}
\hline \multirow[t]{2}{*}{ Category } & \multicolumn{2}{|c|}{ No. $\times 1000(95 \%$ UI $)$} & \multicolumn{2}{|c|}{ ASR per $100,000(95 \%$ UI $)$} & \multirow{2}{*}{$\begin{array}{c}\text { EAPC \% }(95 \% \mathrm{CI}) \\
1990-2017\end{array}$} \\
\hline & 1990 & 2017 & 1990 & 2017 & \\
\hline \multirow[t]{2}{*}{ Overall } & 9285.18 & 15097.64 & 2.91 & 2.78 & -0.347 \\
\hline & $(9876.15,8602.70)$ & $(16663.54,13248.09)$ & $(3.09,2.70)$ & $(2.44,3.07)$ & $(-0.493,-0.200)$ \\
\hline \multicolumn{6}{|l|}{ Sex } \\
\hline \multirow[t]{2}{*}{ Male } & 7281.27 & 12586.16 & 5.30 & 5.07 & -0.340 \\
\hline & $(7730.07,6754.50)$ & $(13927.15,10928.60)$ & $(5.63,4.91)$ & $(4.40,5.61)$ & $(-0.492,-0.188)$ \\
\hline \multirow[t]{2}{*}{ Female } & 2003.91 & 2511.48 & 1.06 & 0.83 & -1.116 \\
\hline & $(2301.55,1682.34)$ & $(3020.14,1960.36)$ & $(1.21,0.89)$ & $(0.65,0.99)$ & $(-1.267,-0.965)$ \\
\hline \multicolumn{6}{|l|}{$\begin{array}{l}\text { Risk } \\
\text { factors }\end{array}$} \\
\hline \multirow{2}{*}{ Smoking } & 6420.32 & 7493.44 & 2.00 & 1.37 & -1.613 \\
\hline & $(5740.24,7024.07)$ & $(6521.15,8504.44)$ & $(1.80,2.18)$ & $(1.19,1.55)$ & $(-1.825,-1.400)$ \\
\hline \multirow{2}{*}{$\begin{array}{l}\text { Alcohol } \\
\text { use }\end{array}$} & 3827.03 & 6784.80 & 1.23 & 1.27 & 0.055 \\
\hline & $(2730.28,4847.96)$ & $(4582.50,8831.67)$ & $(0.89,1.55)$ & $(0.86,1.64)$ & $(-0.094,0.205)$ \\
\hline High & 3153.58 & 6981.15 & 1.00 & 1.29 & 0.777 \\
\hline $\begin{array}{l}\text { mass } \\
\text { index }\end{array}$ & $(1062.05,5545.67)$ & $(2429.76,11398.00)$ & $(0.34,1.75)$ & $(0.45,2.11)$ & $(0.560,0.993)$ \\
\hline \multirow{2}{*}{$\begin{array}{l}\text { Diet low } \\
\text { in fruits }\end{array}$} & 1507.13 & 2920.67 & 0.47 & 0.54 & 0.547 \\
\hline & $(308.28,2994.30)$ & $(615.42,5594.33)$ & $(0.10,0.94)$ & $(0.11,1.03)$ & $(0.470,0.624)$ \\
\hline \multirow{2}{*}{$\begin{array}{l}\text { Chewing } \\
\text { tobacco }\end{array}$} & 554.49 & 879.89 & 0.17 & 0.16 & -0.481 \\
\hline & $(321.24,807.54)$ & $(465.46,1362.45)$ & $(0.10,0.25)$ & $(0.09,0.25)$ & $(-0.557,-0.405)$ \\
\hline
\end{tabular}

472 ASR, age-standardized rate; CI, confidence interval; EAPC, estimated annual percentage change; UI, 473 uncertainty interval. 
475 Table 3. DALYs and ASRs of DALYs of esophageal cancer in 1990 and 2017 and temporal trends.

\begin{tabular}{|c|c|c|c|c|c|}
\hline \multirow[t]{2}{*}{ Category } & \multicolumn{2}{|c|}{ Year ×1000 $(95 \%$ UI $)$} & \multicolumn{2}{|c|}{ ASR per $100,000(95 \%$ UI) } & \multirow{2}{*}{$\begin{array}{c}\text { EAPC \% }(95 \% \\
\text { CI })\end{array}$} \\
\hline & 1990 & 2017 & 1990 & 2017 & \\
\hline \multirow[t]{2}{*}{ Overall } & 213467.95 & 330723.23 & 70.92 & 64.18 & -1.238 \\
\hline & $(226157.04,198788.43)$ & $(364856.87,290539.06)$ & $(75.17,66.02)$ & $(70.78,56.44)$ & $(-1.121,-1.355)$ \\
\hline \multicolumn{6}{|l|}{ Sex } \\
\hline \multirow[t]{2}{*}{ Male } & 172728.76 & 281353.02 & 126.79 & 115.36 & -0.576 \\
\hline & $(183291.51,160688.33)$ & $(311060.98,245363.34)$ & $(134.59,117.80)$ & $\begin{array}{c}(127.51 \\
100.63)\end{array}$ & $(-0.441,-0.711)$ \\
\hline \multirow[t]{2}{*}{ Female } & 40739.19 & 49370.21 & 23.91 & 18.04 & -0.563 \\
\hline & $(46360.38,34711.73)$ & $(58521.31,39108.44)$ & $(27.11,20.44)$ & $(21.33,14.34)$ & $(-0.422,-0.703)$ \\
\hline \multicolumn{6}{|l|}{$\begin{array}{l}\text { Risk } \\
\text { factors }\end{array}$} \\
\hline & 145477.43 & 160233.75 & 47.94 & 30.48 & -1.912 \\
\hline \multicolumn{5}{|l|}{ Smoking } & $(-1.719,-2.105)$ \\
\hline \multirow{2}{*}{$\begin{array}{l}\text { Alcohol } \\
\text { use }\end{array}$} & 93814.69 & 156948.51 & 31.84 & 31.01 & -0.197 \\
\hline & $(116931.38,69625.24)$ & $(200340.05,109707.07)$ & $(39.44,23.69)$ & $(39.36,21.96)$ & $(-0.055,-0.338)$ \\
\hline $\begin{array}{l}\text { High } \\
\text { body- } \\
\text { mass } \\
\text { index }\end{array}$ & $(130926.43,25248.13)$ & $(254322.82,53341.17)$ & $(43.73,8.47)$ & $(49.33,10.33)$ & $(0.716,0.304)$ \\
\hline \multirow{2}{*}{$\begin{array}{l}\text { Diet low } \\
\text { in fruits }\end{array}$} & 34341.41 & 62790.16 & 11.42 & 12.26 & 0.280 \\
\hline & $(68026.85,7056.94)$ & $(121273.22,13272.90)$ & $(22.64,2.34)$ & $(23.59,2.61)$ & $(0.360,0.200)$ \\
\hline \multirow[b]{2}{*}{$\begin{array}{l}\text { Chewing } \\
\text { tobacco }\end{array}$} & 12574.38 & 19609.44 & 4.19 & 3.81 & -0.627 \\
\hline & $(18307.86,7217.65)$ & $(30394.92,10350.81)$ & $(6.13,2.39)$ & $(5.91,2.05)$ & $(-0.542,-0.711)$ \\
\hline
\end{tabular}

476 DALYs, mortality and disability adjusted life years; ASR, age-standardized rate; CI, confidence 477 interval; EAPC, estimated annual percentage change; UI, uncertainty interval. 

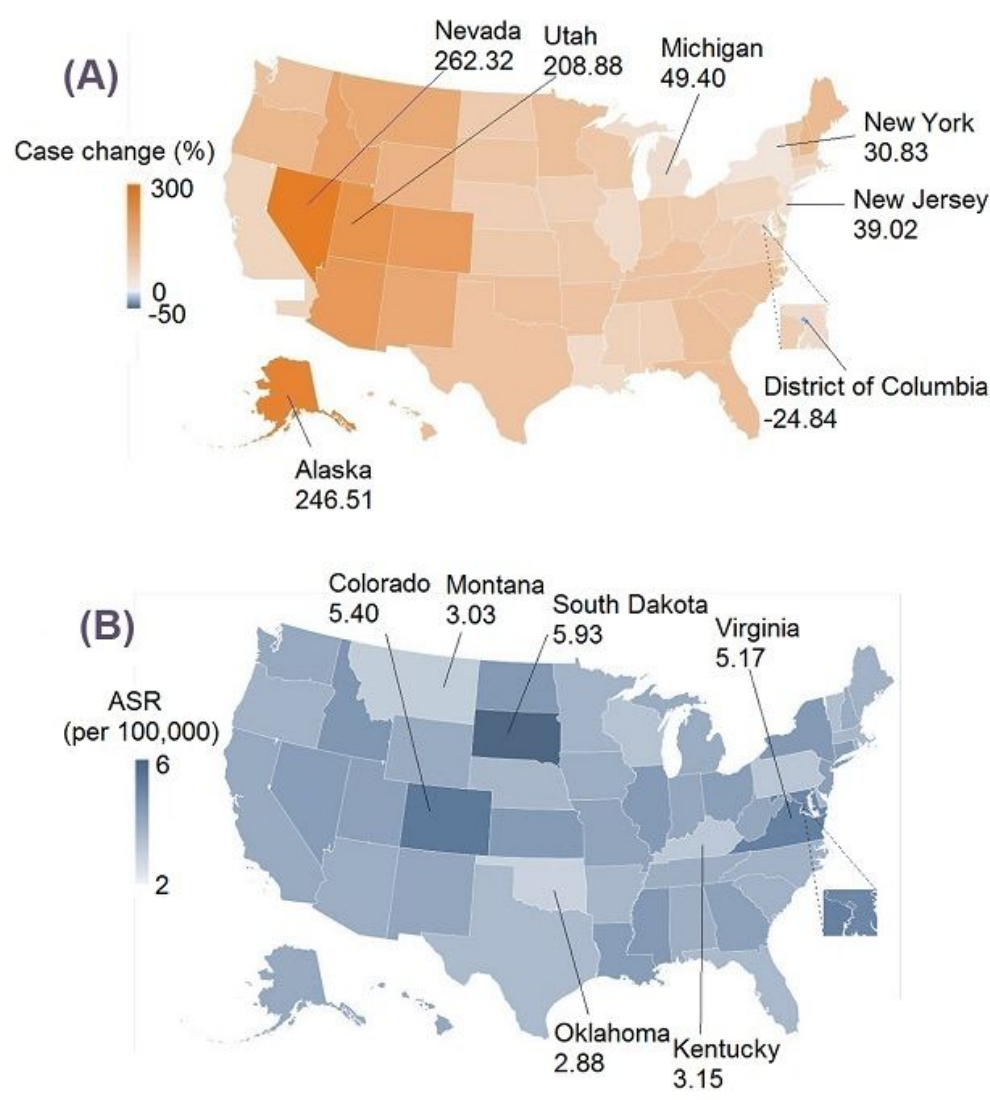

(D)

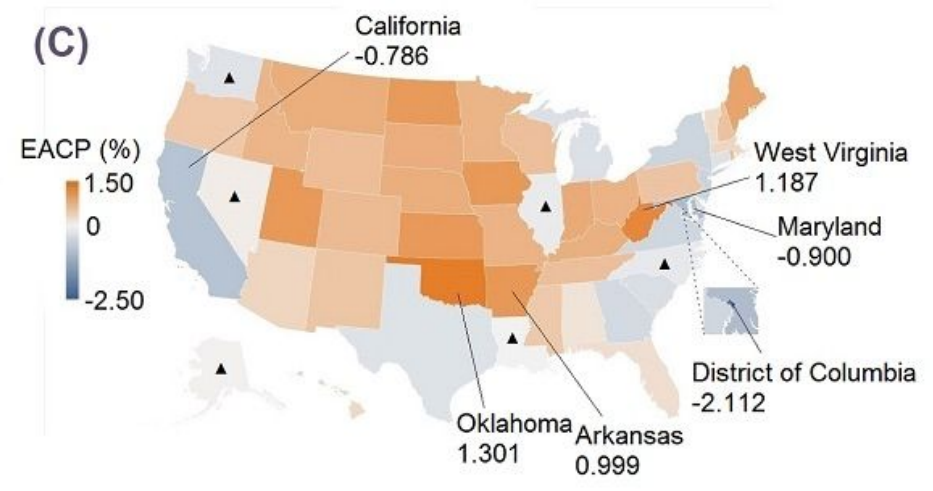

(E)

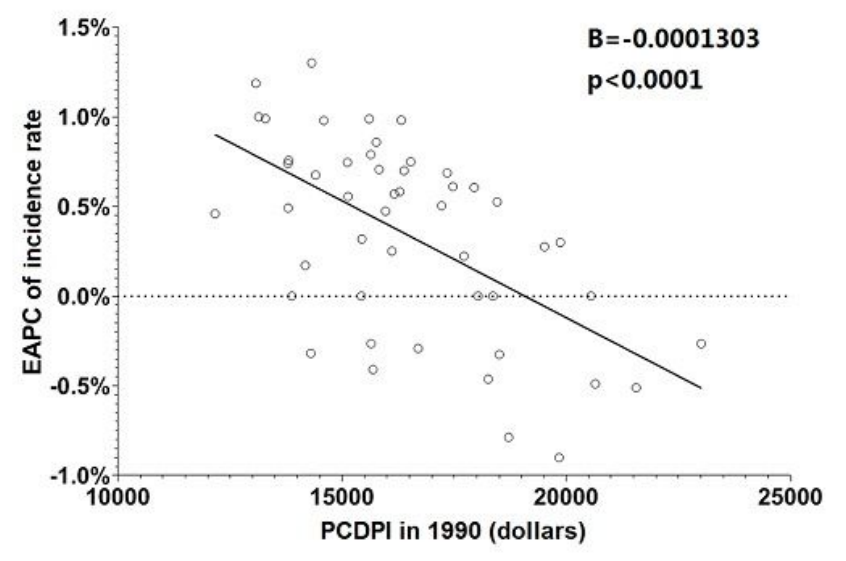

\section{Figure 1}

Maps of parameters of incidence, and the correlation between PCDPI and EAPC. (A) The relative change in new cases of esophageal cancer between 1990 and 2017. (B) The ASR of incidence of esophageal cancer in 2017 (C) The EAPC of ASR of incidence of esophageal cancer from 1990 to 2017. (D)The correlation between PCDPI and ASR of incidence of esophageal cancer. (E) The correlation between PCDPI and EAPC. The B indices and $p$ values presented in (D) and (E) were derived from Pearson correlation analysis. The circles in $(\mathrm{C})$ to $(\mathrm{L})$ represent states. ASR, age-standardized rate; EAPC, estimated annual percentage change. $\mathbb{Q}$, EAPC is not significant. District of Columbia was not included in (D) and (E) because of its outlier high PCDPI. 


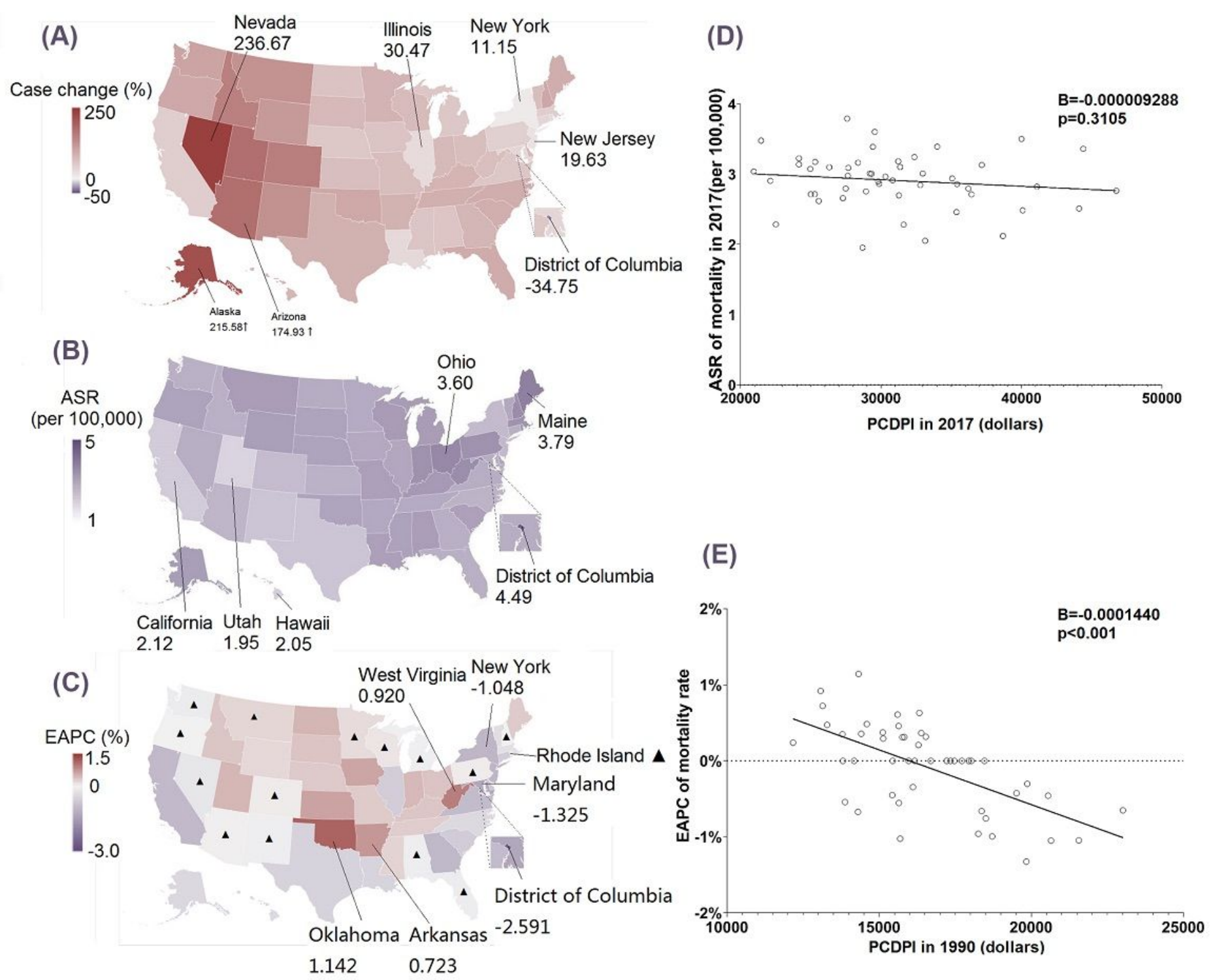

\section{Figure 2}

Maps of parameters of mortality, and the correlation between PCDPI and EAPC. (A) ASRs of mortality of esophageal cancer attributed to different risk factors in different states. (B) EAPC of mortality of esophageal cancer attributed to different risk factors in different states. (C-G) The correlation between PCDPI and ASR of mortality of esophageal cancer attributed to certain risk factor and its EAPC. (H-L) The correlation between PCDPI and EAPC of mortality of esophageal cancer attributed to certain risk factor and its EAPC. ASR, age-standardized rate; EAPC, estimated annual percentage change. Boxes with slashes mean that EAPC is not significant. The circles represent states. The $B$ indices and $p$ values were derived from Pearson correlation analysis. ASR, age-standardized rate; EAPC, estimated annual percentage change. District of Columbia was not included in $(C)$ and $(L)$ because of its outlier high PCDPI. 

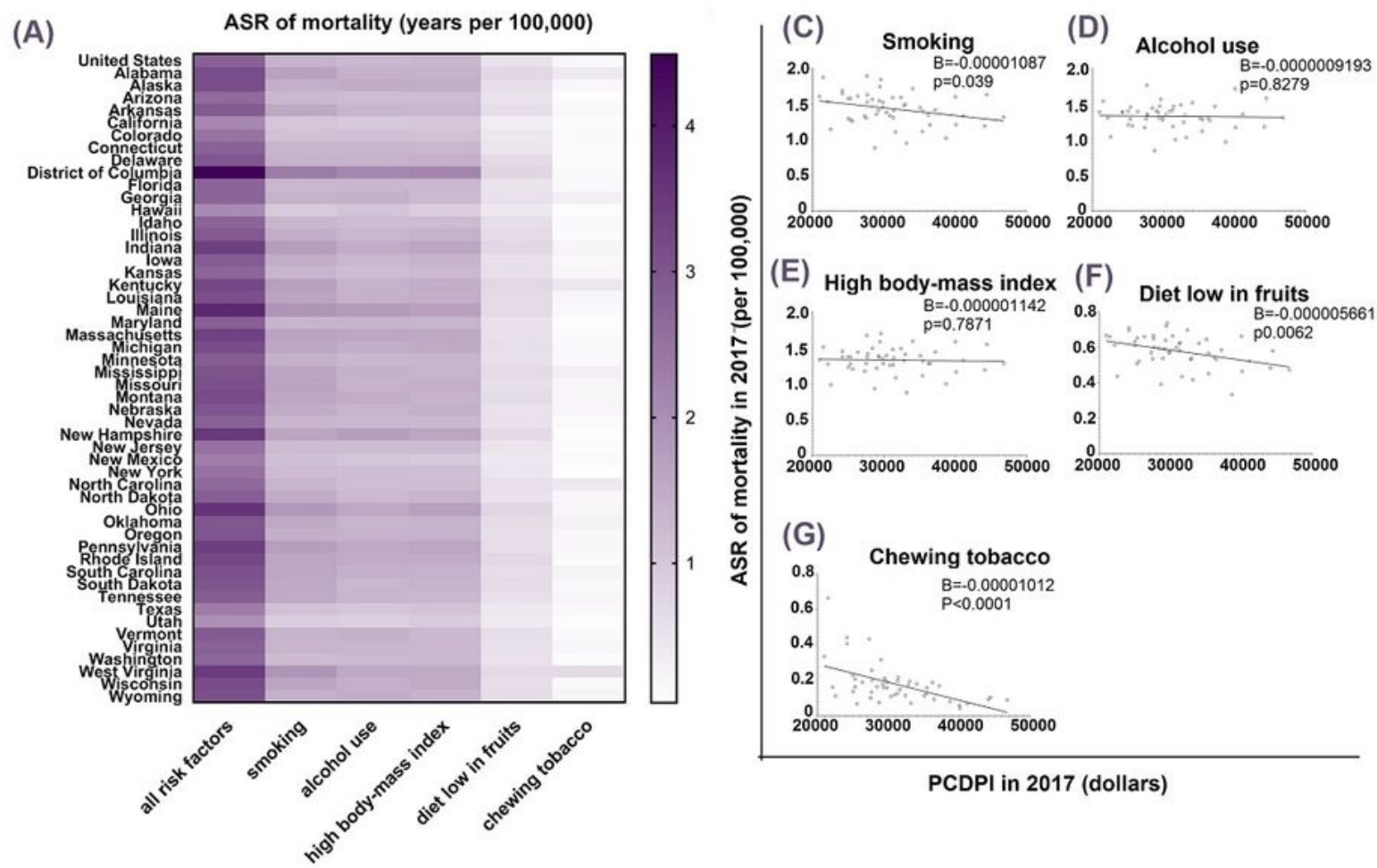

(B)

EACP of mortality rate
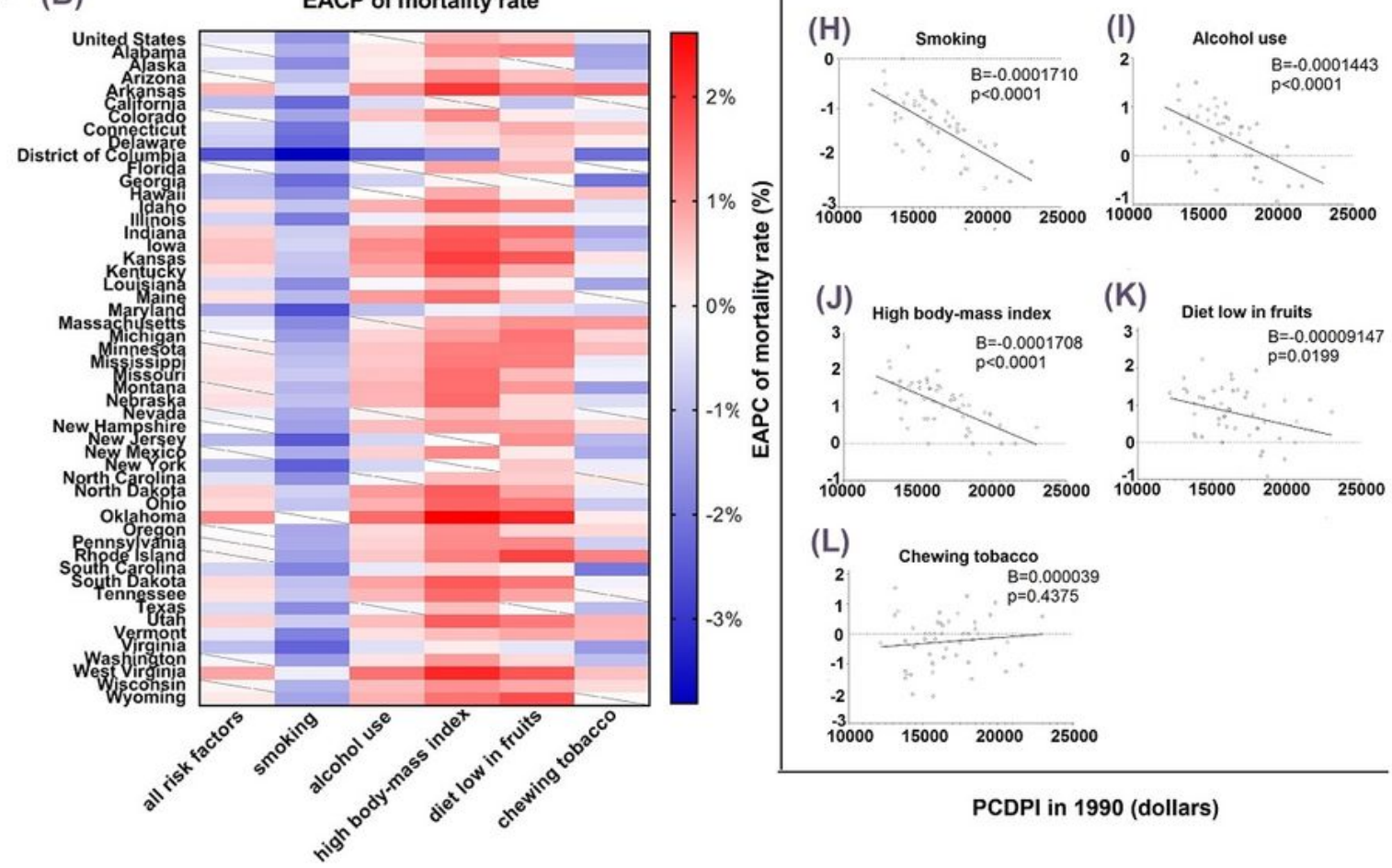

(L) Chewing tobacco

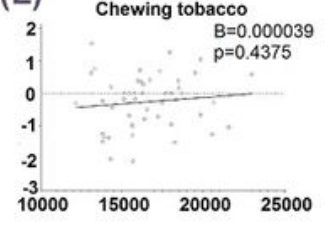

PCDPI in 1990 (dollars)

\section{Figure 3}

Heat map of EAPCs of mortality, and the correlation between PCDPI and EAPC. (A) ASRs of mortality of esophageal cancer attributed to different risk factors in different states. (B) EAPC of mortality of esophageal cancer attributed to different risk factors in different states. (C-G) The correlation between PCDPI and ASR of mortality of esophageal cancer attributed to certain risk factor and its EAPC. (H-L) The correlation between PCDPI and EAPC of mortality of esophageal cancer attributed to certain risk factor 
and its EAPC. ASR, age-standardized rate; EAPC, estimated annual percentage change. Boxes with slashes mean that EAPC is not significant. The circles represent states. The $B$ indices and $p$ values were derived from Pearson correlation analysis. ASR, age-standardized rate; EAPC, estimated annual percentage change. District of Columbia was not included in (C) and ( $L$ ) because of its outlier high PCDPI.

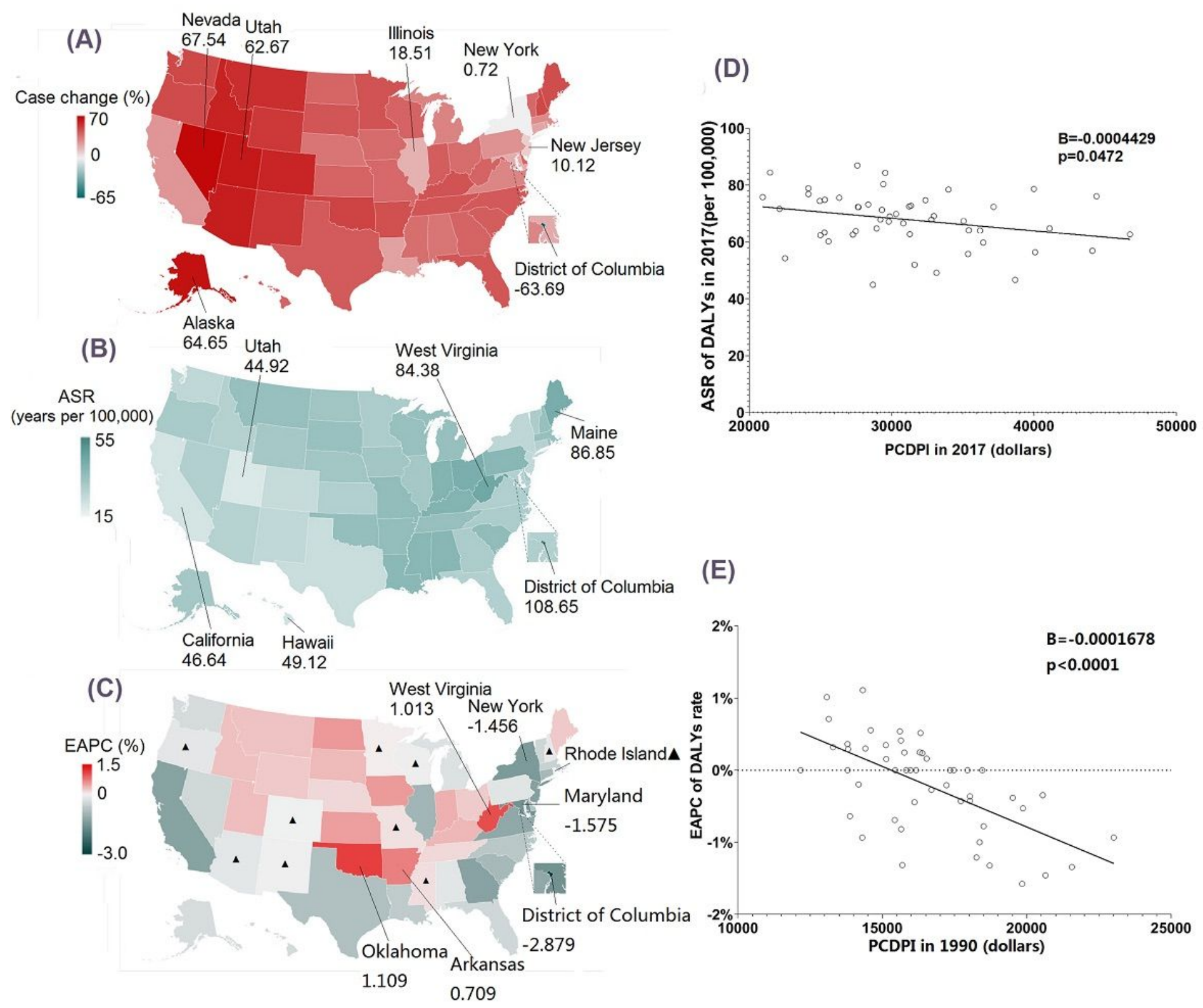

\section{Figure 4}

Maps of parameters of DALYs, and the correlation between PCDPI and EAPC. (A) The relative change in DALYs of esophageal cancer between 1990 and 2017. (B) The ASR of DALYs of esophageal cancer in 2017. (C) The EAPC of ASR of DALYs of esophageal cancer from 1990 to 2017. (D) The correlation between PCDPI and ASR of DALYs of esophageal cancer. (E) The correlation between PCDPI and EAPC. The $B$ indices and $p$ values presented in (D) and (E) were derived from Pearson correlation analysis. The circles in (C) to $(L)$ represent states. DALYs, mortality and disability adjusted life years; ASR, age- 
standardized rate; EAPC, estimated annual percentage change. 囚, EAPC is not significant. District of Columbia was not included in (D) and (E) because of its outlier high PCDPI.
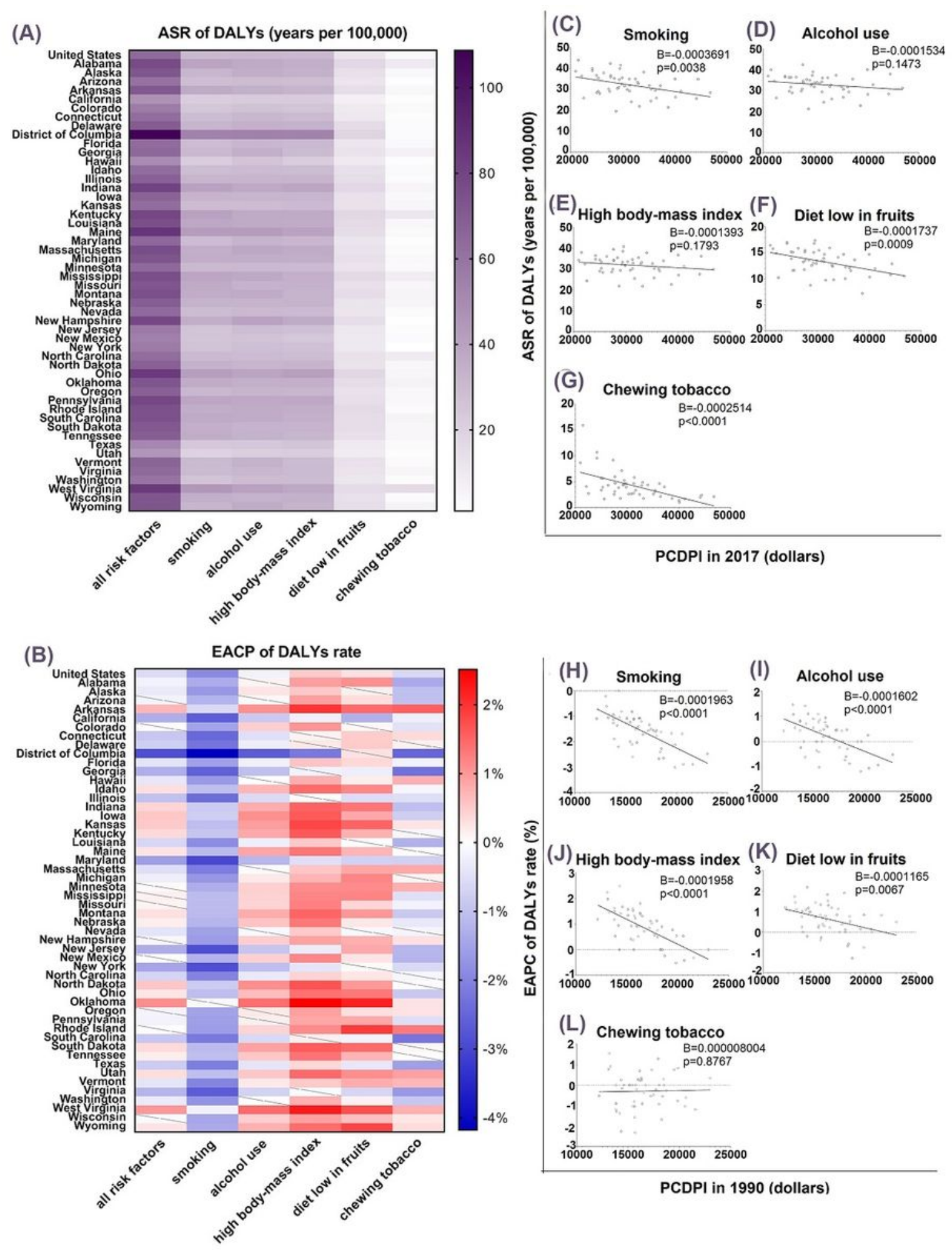

Figure 5

Heat map of EAPCs of DALYs, and the correlation between PCDPI and EAPC. (A) ASRs of DALYs of esophageal cancer attributed to different risk factors in different states. (B) EAPC of DALYs of esophageal cancer attributed to different risk factors in different states. (C-G) The correlation between 
PCDPI and ASR of DALYs of esophageal cancer attributed to certain risk factor and its EAPC. $(\mathrm{H}-\mathrm{L})$ The correlation between PCDPI and EAPC of DALYs rate of esophageal cancer attributed to certain risk factor and its EAPC. ASR, age-standardized rate; EAPC, estimated annual percentage change. Boxes with slashes mean that EAPC is not significant. The circles represent states. The $B$ indices and $p$ values were derived from Pearson correlation analysis. DALYs, mortality and disability adjusted life years; ASR, agestandardized rate; EAPC, estimated annual percentage change. District of Columbia was not included in (C) to (L) because of its outlier high PCDPI. 